\title{
Sub-GHz Inductive Power Transmission from Helical Coils for Implanted Medical Devices
}

\author{
A. $\operatorname{Diet}^{1}$, S. Koulouridis ${ }^{1,2}$, Y. Le Bihan ${ }^{1}$, Q.-T. Luu ${ }^{1}$, O. Meyer $^{1}$, L. Pichon ${ }^{1}$, M. Biancheri-Astier ${ }^{1}$ \\ ${ }^{1,}$ GeePs, UMR CNRS 8507, Université Paris-Saclay, Sorbonne Universités. \\ 11 rue Joliot-Curie, Plateau de Moulon 91192 Gif-sur-Yvette, France \\ ${ }^{2}$ School of Electrical and Computer Engineering, University of Patras, Patras, Greece. \\ antoine.diet@geeps.centralesupelec.fr, koulouridis@g.upatras.gr, yann.le-bihan@geeps.centralesupelec.fr
}

\begin{abstract}
This paper presents measurements and simulations for small helical coils that provide inductive power transfer for implanted medical devices (IMDs) in sub-GHz region. In such context, the coils need be very small to be implanted within devices, or even to be used as an alternate solution combined with UHF antennas. The influence of the body and the mandatory small size of the coils bring technological challenges concerning efficiency and measurements accuracy which must validate our simulations. In this paper, a deembedding technique is used to take into account the dedicated test fixture, and further work will investigate the electromagnetic influence of a liquid phantom, modeling the muscle tissue.
\end{abstract}

Keywords-IMD, antennas, WPT, coils, measurements

\section{INTRODUCTION}

Implantable Medical Devices (IMDs) offer several advantages for diagnostic and therapeutic purposes including sensing, stimulation, and drug delivery. Since the first implementation of pacemakers in 1958, numerous researches have been conducted around the world to apply state-of-the-art techniques to the implantable medical devices (IMDs) industry. In order to design smaller IMDs and to reduce potential interaction with the human body, a device with or without embedded rechargeable batteries is desired.

The topic of IMDs is of great interest for electrical engineering scientific community since their wireless powering and communication ability are difficult equations. Additionally, the technical solution proposed for power transfer (as in WPT and RF harvesting topics) and wireless communication (NFC or Far Field) concern two key cases: inbody to in-body or out-of-body to in-body power transmission $[1][2][3]$. As shown in Figure 1, the body has a complex permittivity which can strongly influence the designs of the antennas. Frequencies investigated for powering and communicating with IMDs vary from HF to UHF, because of large size devices at lowest frequencies in one hand and high losses at higher frequencies inside the body in the other hand. The design of antennas for IMDs has been extensively investigated like PIFA antennas [2][3] and the challenge lies nowadays on technically combining different RF modes in far field or near field for powering and communicating.
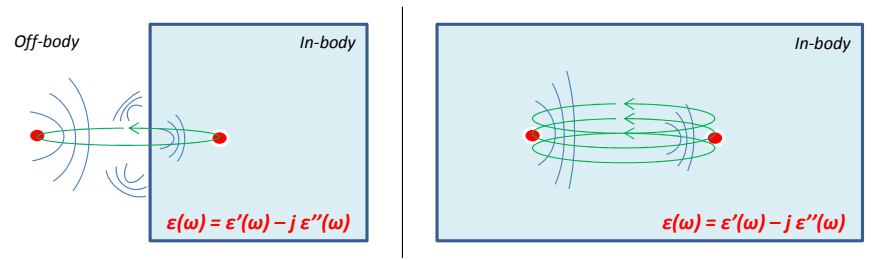

Figure 1: the two cases of IMDs RF powering or communication

In this paper we focus on the feasibility of a very small coil which can transfer energy at low distance and could be used in one of the two cases in Figure 1. This could efficiently further support current IMD for which only radiating RF mode is used. The subject has been initially studied in [3] where a coil was embedded into miniaturized PIFA for IMDs. The design of such a coil will be influenced by tissues dielectric properties [4][5][6]. Also, their size has to be extremely small if implanted and figures of merit on the efficiency of the combined system [6][7] of combined system (coil-antenna) should be defined. In the second part of this paper we will present simulation results considering an arm for biological tissue. In the third part of the paper we described the design and measurements of small coils, with a diameter of 2,5 $\mathrm{mm}$, at a distance of $10 \mathrm{~mm}$, including the mandatory 2-port deembedding techniques (since coils are smaller than SMA connectors and are highly affected by them) [8][9][10]. Finally, the last part concludes on further and current works concerning measurement deembedding in phantoms, used for modeling the muscle.

\section{INDUCTIVE POWERING SIMULATION}

The transmission of a signal between two coils is investigated in the case of a helical coil for the receiver and a loop at the transmitter, in the presence of a muscle tissue under CST microwaves studio, as seen in Figure 2. 


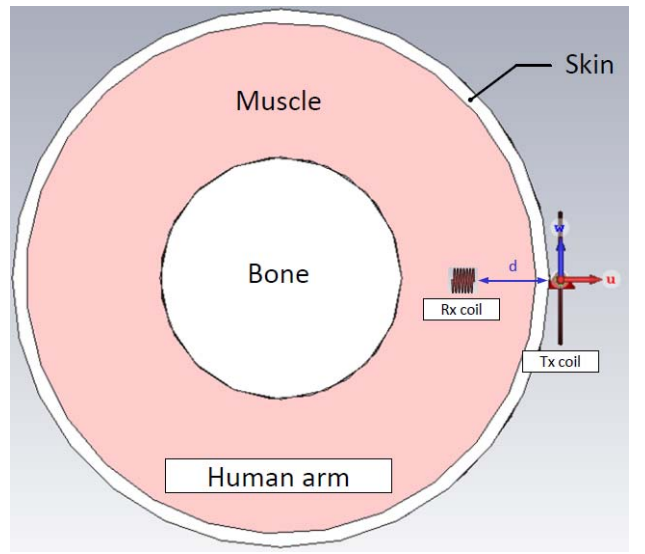

Figure 2: RF link simulation between coils inside muscle tissue

In order to analyze the efficiency of this design and to be able to determine the optimal frequency due to the electromagnetic influence of the body, we used the same figure of merit, PTE, reported by Ahn et al. in [7], see (1).

$$
P T E \approx \frac{\left|Z_{21}\right|^{2}}{\left|\operatorname{Im}\left(Z_{11}\right) \operatorname{Im}\left(Z_{22}\right)\right|} \frac{\left|\operatorname{Im}\left(Z_{11}\right)\right|}{R_{11}} \frac{\left|\operatorname{Im}\left(Z_{22}\right)\right|}{\left(R_{22}+R_{L}\right)} \frac{R_{L}}{\left(R_{22}+R_{L}\right)}
$$

The simulation and computing of PTE is done in function of the size (radius) of the transmitter loop in presence or not of the tissue. At a fixed distance of $12 \mathrm{~mm}$, the receiver coil is a helix of $1 \mathrm{~mm}$ height, with a diameter of $1 \mathrm{~mm}$ and has 7 turns. Results are shown in Figure 3 for a coil of $24 \mathrm{~mm}$ in diameter.

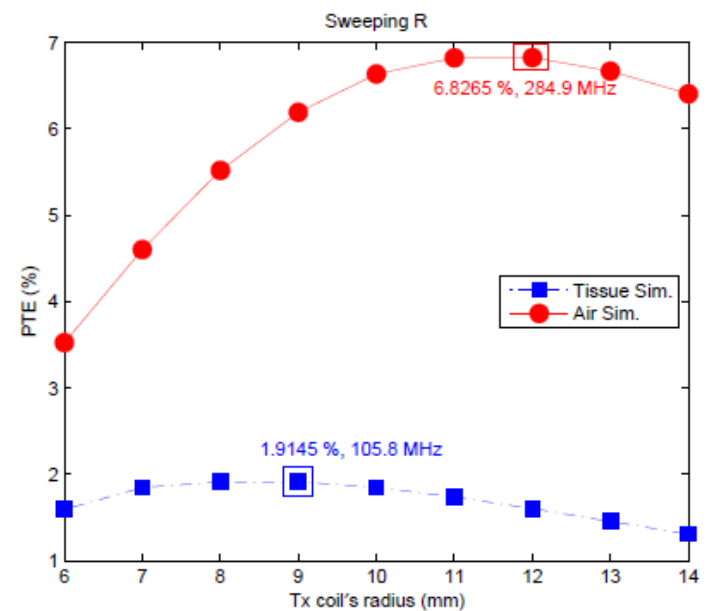

Figure 3 : PTE simulation results in CST for $24 \mathrm{~mm}$-diameter Tx coil radii, in presence or not of the muscle tissue influence.

The simulation result in Figure 3 shows that the presence of the body tissue shifts towards low frequencies the optimal value of power transfer efficiency (i. e. the optimal frequency of operation) and lowers it due to additional losses. Moreover, the design of coils should be investigated by optimizing the shapes of the coil and avoid the presence of a self-resonance in the HF to UHF band of interest (3-300 MHz). In the following part, we designed small identical helical coils for investigating the RF link at such frequencies and verify measurement process before moving to more complex scenarios.

\section{TESTS WITH SMALL COILS}

The design of small coils with a diameter of $2.5 \mathrm{~mm}$ and height of $1 \mathrm{~mm}$, are made with thin copper wire and fixed on nylon screws, as shown in Figure 4. Due to the small size of the helical coils, we used a test fixture with PCB in which the connector are at a distance of $2 \mathrm{~cm}$ from the coils in order to limit the influence of the metal on the electromagnetic near field generated by the coils, as shown in Figure 4 .

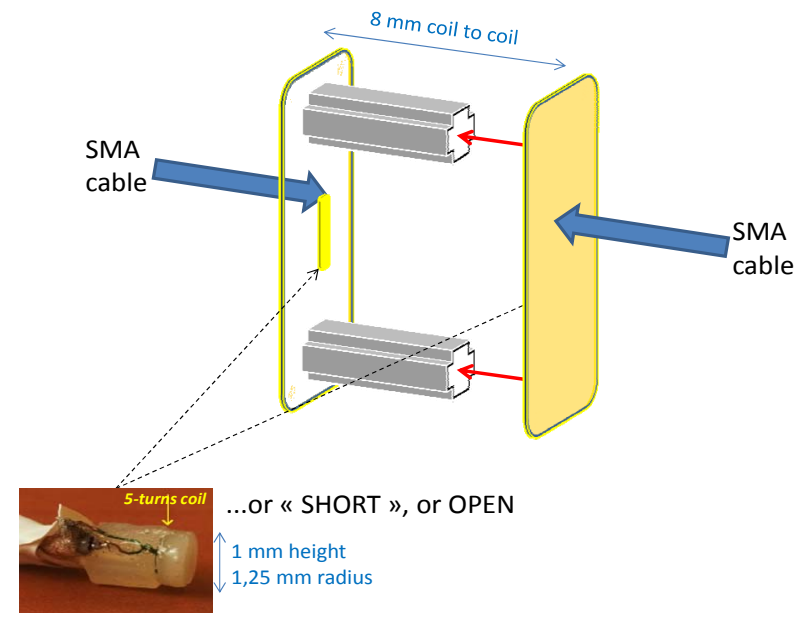

Figure 4: test fixture for the small coils

The test fixture used will drive us to use a deembedding technique adapted to the unavoidable presence of PCB and lines in HF to UHF frequencies. Some matrix techniques of deembedding are presented in [9][10] for transistor measurements and can be fruitfully used in our case. One method is graphically synthesized in Figure 5 and needs the additional design and measurements of 2-ports "short" and "open" configurations with the same test fixture to be able to compute the matrix equation on admittance parameters.

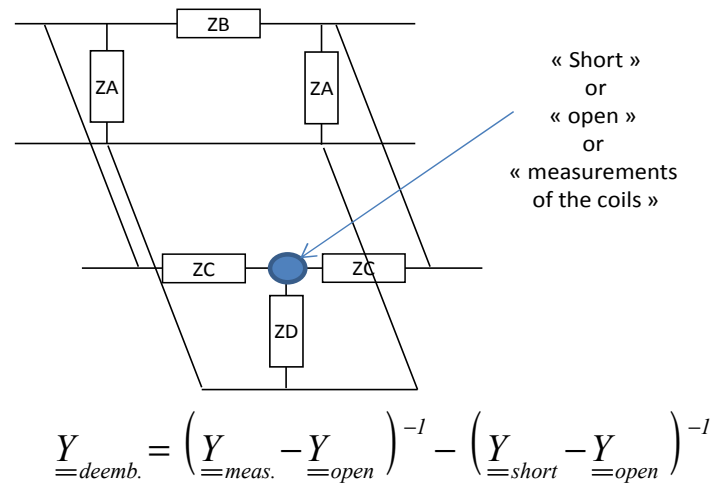

Figure 5 : Extraction of parallel and series parasitic elements by using 2ports "short" and "open" configurations with the test fixture.

Measurements are performed with a Vector Network Analyzer and S parameters are deembedded and converted into 
admittance and impedance parameters under Matlab. An analytical evaluation of the self-inductance of the helical coils is given by Piri et al. in [8], and reported in (2) where $\mathrm{K}(\mathrm{k})$ and $\mathrm{E}(\mathrm{k})$ are the complete elliptic integrals of the first and second kind, respectively.

$$
L=\frac{8}{3} \mu_{0} R^{3} \frac{N^{2}}{h^{2}}\left[\frac{1}{k}\left(\frac{1-k^{2}}{k^{2}}(K(k)-E(k)+E(k))\right)-1\right]
$$

Figure 6 presents the deembedded measurements results for port 1 and port 2. As can be seen in this figure, the measurements deembedded are in the range of the analytical expression with a tolerance of $10 \%$ under $300 \mathrm{MHz}$ (upper limit of VHF). In UHF, measurements are not of good accuracy due to the potential self-resonances of coils and or parasitic elements of the test fixture and connectors soldering, issues present even with the deembedding techniques.

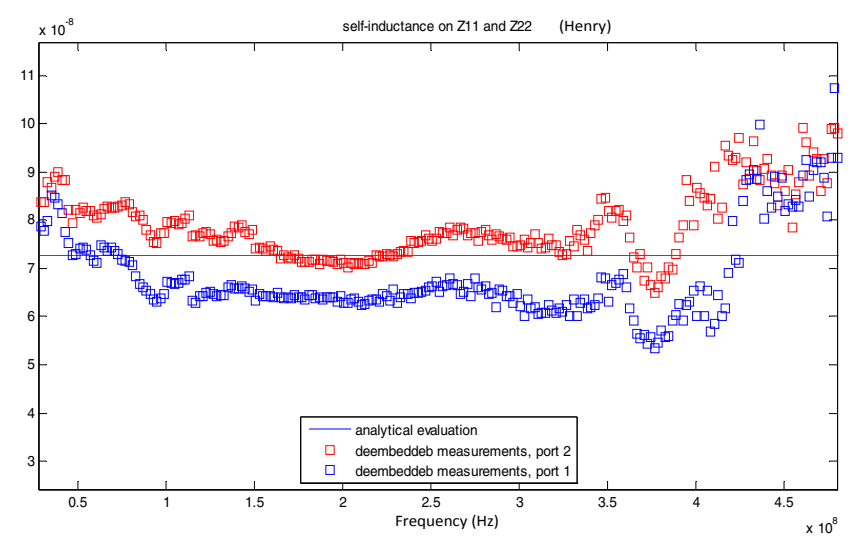

Figure 6: Comparison of self-inductance extraction from the measurements and analytical expression given by (2)

\section{CURRENT WORK AND CONCLUSION}

The measurements of the previous part show an interesting matching for self-inductance evaluation under $300 \mathrm{MHz}$ with a simple calibration test fixture. Some parasitics are still present after deembedding and we need to improve the design of the test fixture to be able to evaluate the whole impedance matrix of the 2 coils system.

The design of the coils in the presence of a biological tissue can be fruitfully helped with measurements of the coil inside a phantom made with Triton $X$, water and salt. This phantom is shown in Figure 7, in which its relative permittivity and conductivity are also given in function of the frequency. Next step of the design process is to accurately deembed and resimulate to match the characteristics of the medium for optimization of the small coils.
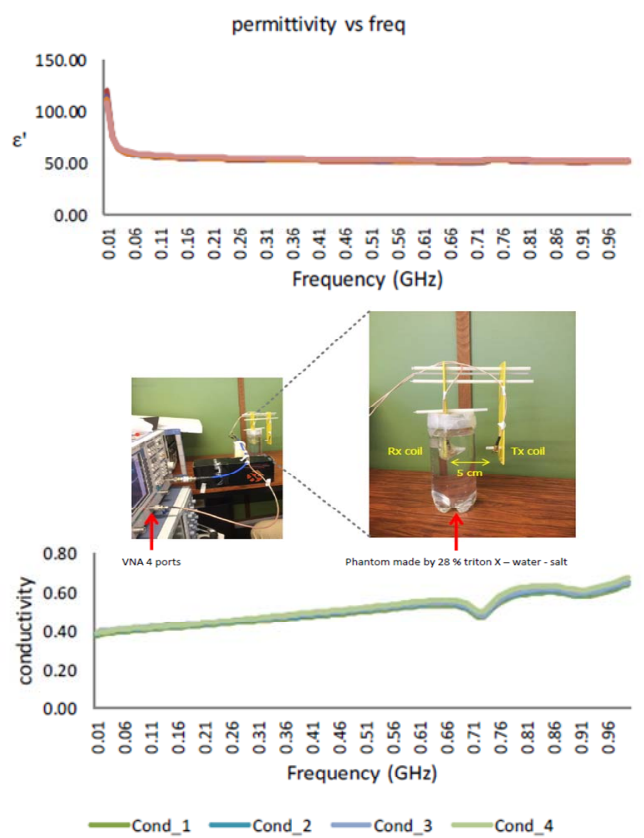

Figure 7 : Muscle liquid phantom used in the laboratory and its measured electromagnetic properties

\section{ACKNOWLEDGMENT}

The authors want to acknowledge Richard Beljio and Michel Police for their help in the design of the test fixture.

\section{REFERENCES}

[1] Y.-H. Joung, Development of Implantable Medical Devices: From an Engineering Perspective. International Neurourology Journal, vol. 17, no. 3, pp. 98-106, 2013.

[2] S. Bakogianni and S. Koulouridis. Design of a Novel Miniature Implantable Rectenna for In-Body Medical Devices Power Support, 10th European Conference on Antennas and Propagation EuCAP 2016.

[3] S. Koulouridis, S. Bakogianni, A. Diet, Y. L. Bihan, and L. Pichon, Investigation of Efficient Wireless Charging for Deep Implanted Medical Devices in IEEE Symposium on Antennas and Propagation (AP-S) and USNC-URSI Radio Science Meeting, pp. 1045-1046, 2016.

[4] S. Kim, S. Member, J. S. Ho, S. Member, A. S. Y. Poon, and S. Member, Wireless Power Transfer to Miniature Implants : Transmitter Optimization, in International Symposium on Antennas and Propagation; USNC/USRI National Radio Science Meeting, 2011.

[5] Y. P. Lin and K. T. Tang, An Inductive Power and Data Telemetry Subsystem with Fast Transient Low Dropout Regulator for Biomedical Implants, IEEE Transactions on Biomedical Circuits and Systems, vol. 10, no. 2, pp. 435-444, 2015.

[6] X. Zhang, R. F. Xue, K. W. Cheng, J. H. Cheong, C. K. Ho, L. Yao, C. $\mathrm{He}$, and M. Je, Design of high-efficiency inductive power transfer coils for biomedical implants, 2013 IEEE MTT-S International Microwave Workshop Series on RF.

[7] D. Ahn et al., Optimal Design of Wireless Power Transmission Links for Millimeter-Sized Biomedical Implants, IEEE Trans. Biomed. Circuits Syst., pp. 1-13, 2014.

[8] M. Piri, V. Jaros, and M. Frivaldsky, Verification of a mutual inductance calculation between two helical coils, in 16th International Scientific Conference on Electric Power Engineering (EPE 2015), pp. 0-5, 2015.

[9] Luuk F. Tiemeijer and Ramon J. Havens, A calibrated Lumped-Element De-Embedding Technique for On-Wafer RF Characterization of HighQuality Inductors and High-Speed Transistors. IEEE transactions on electron Devices, Vol 50, n 3 , march 2003.

[10] M. Koolen, J. Geelen, and M. Versleijen, An improved de-embedding technique for on-wafer high-frequency characterization, Proceedings of the 1991 Bipolar Circuits and Technology Meeting, pp. 188-191, 1991. 